\title{
De novo transcriptome sequencing of radish (Raphanus sativus L.) and analysis of major genes involved in glucosinolate metabolism
}

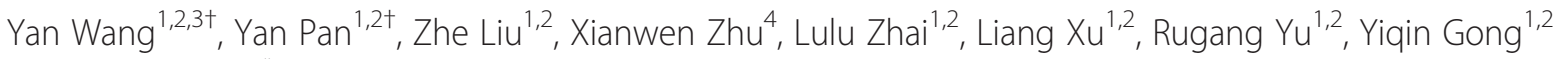
and Liwang Liu ${ }^{1,2^{*}}$

\begin{abstract}
Background: Radish (Raphanus sativus L.), is an important root vegetable crop worldwide. Glucosinolates in the fleshy taproot significantly affect the flavor and nutritional quality of radish. However, little is known about the molecular mechanisms underlying glucosinolate metabolism in radish taproots. The limited availability of radish genomic information has greatly hindered functional genomic analysis and molecular breeding in radish.

Results: In this study, a high-throughput, large-scale RNA sequencing technology was employed to characterize the de novo transcriptome of radish roots at different stages of development. Approximately 66.11 million paired-end reads representing 73,084 unigenes with a N50 length of 1,095 bp, and a total length of $55.73 \mathrm{Mb}$ were obtained. Comparison with the publicly available protein database indicates that a total of 67,305 (about $92.09 \%$ of the

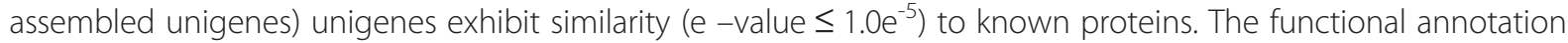
and classification including Gene Ontology (GO), Clusters of Orthologous Group (COG) and Kyoto Encyclopedia of Genes and Genomes (KEGG) analysis revealed that the main activated genes in radish taproots are predominately involved in basic physiological and metabolic processes, biosynthesis of secondary metabolite pathways, signal transduction mechanisms and other cellular components and molecular function related terms. The majority of the genes encoding enzymes involved in glucosinolate (GS) metabolism and regulation pathways were identified in the unigene dataset by targeted searches of their annotations. A number of candidate radish genes in the glucosinolate metabolism related pathways were also discovered, from which, eight genes were validated by T-A cloning and sequencing while four were validated by quantitative RT-PCR expression profiling.

Conclusions: The ensuing transcriptome dataset provides a comprehensive sequence resource for molecular genetics research in radish. It will serve as an important public information platform to further understanding of the molecular mechanisms involved in biosynthesis and metabolism of the related nutritional and flavor components during taproot formation in radish.
\end{abstract}

Keywords: Radish, De novo assembly, RNA-Seq, Transcriptome, Glucosinolate metabolic pathways

\footnotetext{
*Correspondence: nauliulw@njau.edu.cn

${ }^{\dagger}$ Equal contributors

${ }^{1}$ National Key Laboratory of Crop Genetics and Germplasm Enhancement,

College of Horticulture, Nanjing Agricultural University, Nanjing 210095, P.R.

China

${ }^{2}$ Engineering Research Center of Horticultural Crop Germplasm

Enhancement and Utilization, Ministry of Education of P.R. China, College of

Horticulture, Nanjing Agricultural University, Nanjing 210095, P.R. China

Full list of author information is available at the end of the article
} 


\section{Background}

Radish (Raphanus sativus L.) is an annual or biennial herb of the Brassicaceae family, and it is an economically important root vegetable crop produced throughout the world $[1,2]$. The edible part of radish is its taproot, which is an excellent source of carbohydrates, dietary fiber, and essential mineral and organic nutrients to human beings [3-5]. Radish roots also contain valuable phytochemicals and have been used for many medicinal purposes [6,7]. For example, the roots are a rich source of glucosinolates (GS) [8]. GS and their breakdown products such as isothiocyanates (ITC) are secondary metabolites widely present in the Brassicaceae family. The ITC contribute to the flavor and taste of the Brassicaceae vegetables as an important ingredient and have anti-carcinogenic properties $[8,9]$.

The formation and development of taproot is a complex morphogenetic process controlled by interactions among genetic, environmental and physiological factors [1,10-12]. Essentially, fleshy root formation is a result of selective expression of related genes. However, the lack of genomic information impedes our understanding of the molecular mechanisms underlying taproot development. Recent analysis of transcript differences between two cDNA libraries from the early and late seedling developmental stages have demonstrated that a set of genes involved in starch and sucrose metabolism, and in phenylpropanoid biosynthesis may be the dominant metabolic pathways during the early stages of taproot formation in radish [13]. This has enabled the mining of genes that are possibly involved in taproot development. However, the molecular mechanisms involved in biosynthesis and metabolism of the related nutritional and flavor components during taproot formation are not well known, especially for many secondary metabolites such as glucosinolates.

Next-generation sequencing (NGS) -based RNA sequencing for transcriptome methods (RNA-seq) allows simultaneous acquisition of sequences for gene discovery as well as transcript identification involved in specific biological processes. This is especially suitable for non-model organisms whose genomic sequences are unknown [14-16]. In recent years, RNA-seq has emerged as a powerful method for discovering and identifying genes involved in biosynthesis of various secondary metabolites, such as, carotenoid biosynthesis in Momordica cochinchinensis [17], cellulose and lignin biosynthesis in Chinese fir [18], tea-specific compounds i.e. flavonoid, theanine and caffeine biosynthesis pathways in tea [19], biosynthesis of flavonoid in Safflower [20], biosynthesis of active ingredients in Salvia miltiorrhiza [21] and biosynthesis of capsaicinoid in chili pepper [22].

Glucosinolate content is a main trait of radish cultivars and is important for flavor formation and nutritional quality of the taproot $[8,9]$. Previous studies mainly focused on developing analysis methods to determine GS content in radish, and also to determine variation in GS composition or content in different cultivars, growing conditions, and growth stages [8,23,24]. Furthermore, three candidate genes for controlling the GS content in radish roots were identified from single nucleotide polymorphism (SNP) markers developed with GS [25]. However, molecular mechanisms underlying GS metabolism in radish still require elucidation, especially for identification of the full set of genes involved in these related pathways.

In the present study, NGS-based Illumina paired-end solexa sequencing platform was employed to characterize the fleshy taproot de novo transcriptome in radish. A large set of radish transcript sequences were obtained to discover the majority of the activated genes involved in radish taproot. The candidate genes involved in the glucosinolate metabolism and regulation were successfully identified in radish. The sequence of representative genes and expression patterns were further validated. The root de novo transcriptome was comprehensively characterized in radish. This would provide a public information platform for understanding the molecular mechanisms involved in the metabolism of nutritional and flavor components during taproot formation, and facilitate the genetic improvement of quality traits in radish molecular breeding programs.

\section{Results and discussion}

Illumina sequencing and de novo assembly of radish root transcriptome

To develop a comprehensive overview of the radish root transcriptome, a cDNA library denoted as 'CKA', prepared from three mixed RNA samples from taproots at different stages of development (seedling, taproot thickening, and mature stages) was subjected to pair-end read (PE) sequencing with the Illumina platform. It has been reported that PE sequencing not only increases the depth of sequencing, but also improve de novo assembly efficiency $[18,26]$. After removing the reads with adaptors, reads with unknown nucleotides larger than $5 \%$ and low quality reads, 66,110,340 clean PE reads consisting of $5,949,930,600$ nucleotides (nt) were obtained with an average GC content of $47.34 \%$ (Table 1). The output was similar to a previous study on radish transcriptome from two root cDNA libraries, which generated a total of 53.6 million and 53.7 million clean reads, respectively [13]. All high-quality clean reads were assembled into 150,455 contigs with an average length of $299 \mathrm{bp}$, and the length distribution of the assembled contigs was as shown in Additional file 1A. The contigs were further joined into 73,084 unigenes with a N50 length of $1095 \mathrm{bp}$, and a total length of $55.73 \mathrm{Mb}$ using paired-end information and gap- 
Table 1 Statistics of output sequencing

\begin{tabular}{cc}
\hline Samples & CKA \\
\hline Total raw reads & $71,947,118$ \\
Total clean reads & $66,110,340$ \\
Total clean nucleotides (nt) & $5,949,930,600$ \\
Q20 percentage & $97.79 \%$ \\
N percentage & $0.00 \%$ \\
GC percentage & $47.34 \%$ \\
\hline
\end{tabular}

filling process (Table 2). Majority of the unigenes ranged from 300 to $1500 \mathrm{bp}$, and accounted for $88.30 \%$ of all unigenes $(64,418)$ (Additional file 1B).

\section{Functional annotation and classification of the assembled unigenes}

In total, 67,305 (92.09\% of all unigenes) unigenes significantly matched a sequence in at least one of the public databases including NCBI non-redundant protein $(\mathrm{Nr})$, Gene Ontology (GO), Clusters of Orthologous Groups (COGs), Swiss-Prot protein and the Kyoto Encyclopedia of Genes and Genomes (KEGG) (Table 3). The rate of annotated unigenes was higher than the range of previously studies in other non-model species $(73.6 \%$ in blueberry, $58 \%$ in safflower flowers and $58.01 \%$ in Chinese fir), indicating their integrity and the relatively conserved functions of the assembled transcript sequences in radish $[18,20,27]$. The size distribution of the BLAST-aligned coding sequence (CDS) and predicted proteins are shown in Figure 1A, B, respectively. The remaining $7.91 \%$ of unigenes $(5,779)$ that did not match sequences in the databases were analyzed by ESTScan to predict coding regions. An additional 1,573 unigenes (2.15\%) also showed orientation in the transcriptome coding sequence (Figure 1C, D). The sequences without a homologous hit may represent novel genes specifically expressed in radish root; or they could be attributed to other technical or biological biases, such as assembly parameters. Furthermore, some cDNAs are non-coding, lineage-specific or highly variable, which need to be further verified [27-29].

For the $\mathrm{nr}$ annotations, 61,513 of the unigenes (84.17\%) were found to be matched in the database. Further analysis

Table 2 Statistics of assembly quality

\begin{tabular}{ccc}
\hline & Contig & Unigene \\
\hline Total number & 150,455 & 73,084 \\
Total length (nt) & $44,968,854$ & $55,733,722$ \\
Mean length (nt) & 299 & 763 \\
N50 & 458 & 1095 \\
Total consensus sequences & - & 73,084 \\
Distinct clusters & - & 38,040 \\
Distinct singletons & - & 35,044 \\
\hline
\end{tabular}

Table 3 Summary statistics of functional annotation for radish root unigenes in public databases

\begin{tabular}{ccc}
\hline Public protein database & No. of unigene hit & Percentage (\%) \\
\hline NR & 61,513 & 84.17 \\
SwissProt & 38,946 & 53.29 \\
KEGG & 33,567 & 45.93 \\
COG & 19,888 & 27.21 \\
GO & 52,572 & 71.93 \\
ALL & 67,305 & 92.09 \\
\hline
\end{tabular}

of the BLAST data indicated that $57.06 \%$ of the top hits showed strong homology with the E-value $<1.0 \mathrm{e}^{-45}$, while $65.47 \%$ of the matched sequences showed moderate homology with the E-value between $1.0 \mathrm{e}^{-5}$ and $1.0 \mathrm{e}^{-45}$ (Figure 2A). The identity distribution pattern showed that $57.42 \%$ of the sequences had a similarity higher than $80 \%$, while $42.28 \%$ showed similarity between $19 \%$ and $80 \%$ (Figure 2B). The majority of the annotated sequences corresponded to the known nucleotide sequences of plant species, with $45.44 \%, 39.47 \%, 3.41 \%$, $1.98 \%$ and $1.45 \%$ matching with A. lyrata subsp. Lyrata, A. thaliana, Thellungiella halophila, B. napus and $B$. oleracea, respectively (Figure $2 \mathrm{C}$ ). All the top five species with BLAST hits belonged to the Brassicaceae family, implying that the sequences of the radish transcripts obtained in the present study were assembled and annotated properly [30].

GO annotation is an international classification system that can provide standardized vocabulary for assigning functions of the uncharacterized sequences [31]. BLAST2GO program was used to get GO terms for all assembled unigenes and a total of 52,572 unigenes (71.93\% of all the assembled unigenes) were assigned at least one GO term. In many cases, multiple terms were assigned to the same transcript, and all the GO terms were classified into 58 functional groups including biological processes, cellular component, and molecular function at the second level (Figure 3). Among biological processes, transcript sequences assigned to cellular $(39,716)$ and metabolic processes $(37,191)$ were the most abundant. Within the molecular function category, the majority of the GO terms were predominantly assigned to binding $(29,099)$ and catalytic activity $(24,635)$. For cellular components, those assignments were mostly given to cell $(37,809)$ and cell part $(37,803)$. The findings revealed that the main GO classifications involved in the annotated unigenes were responsible for fundamental biological regulation and metabolism. These results were concurrent with a previously reported study of de novo transcriptome analysis in tuberous root of sweet potato [28].

Every protein in the COG database is assumed to be evolved from an ancestor, and the whole database is built on coding proteins with complete genomes as well as 


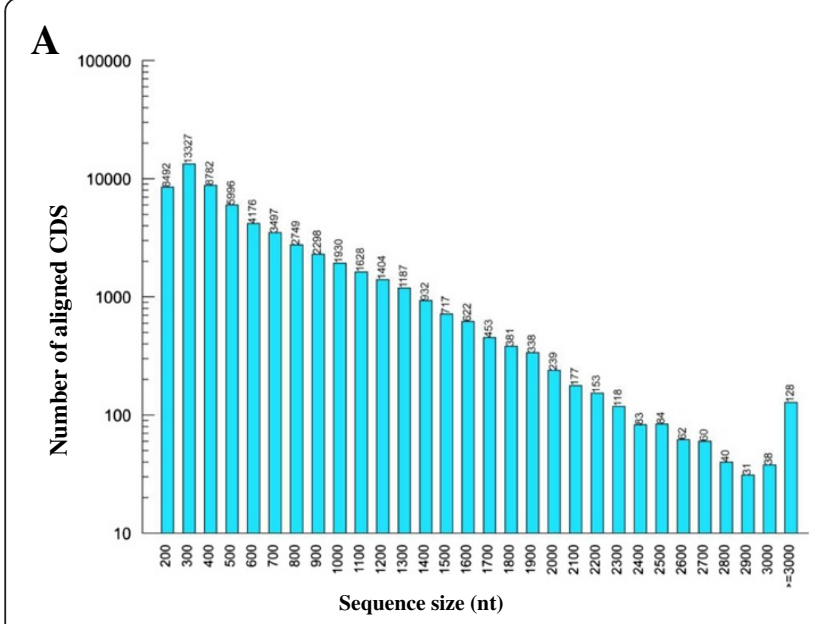

B

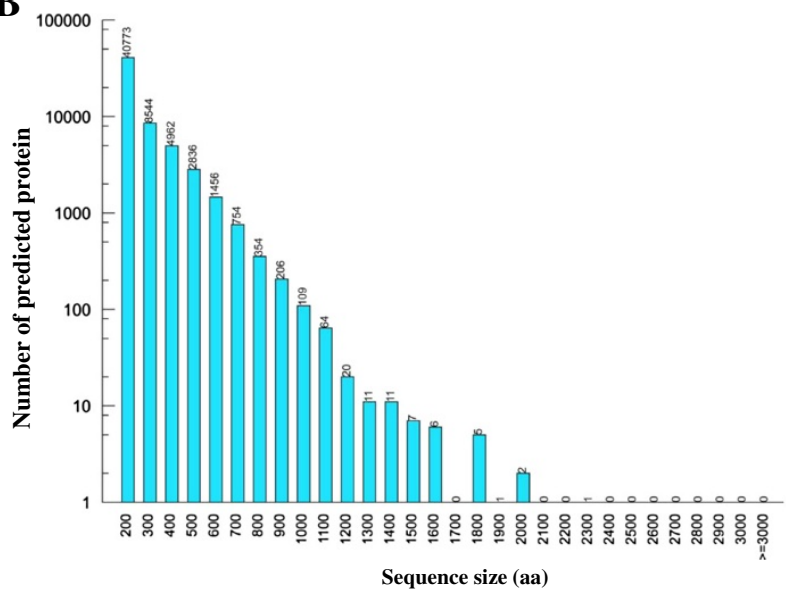

C

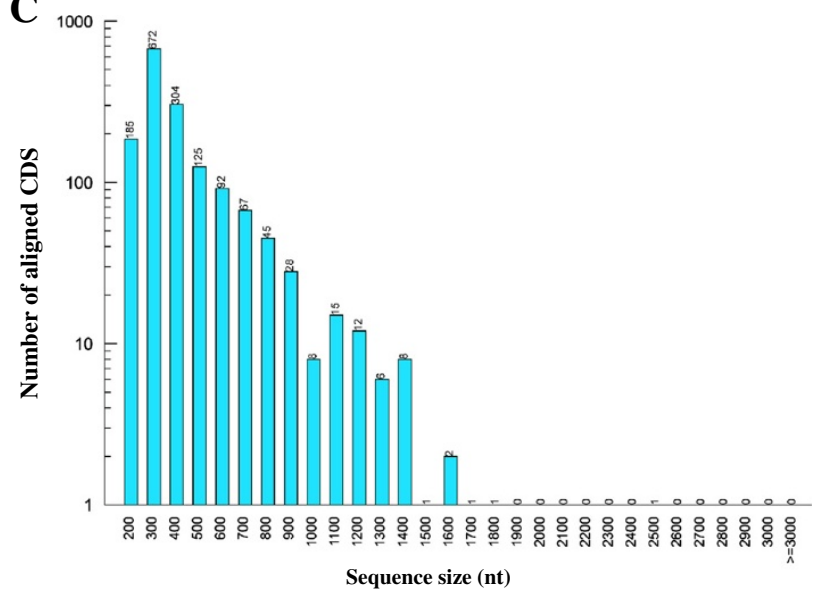

D

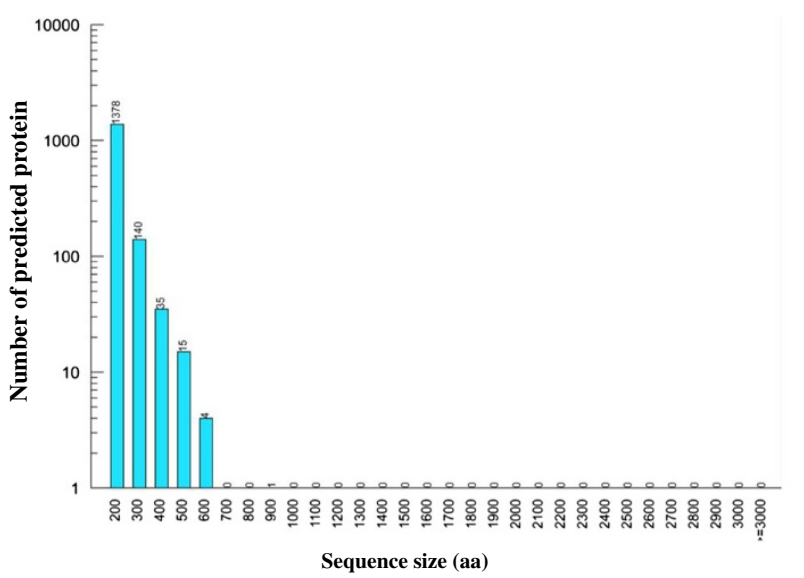

Figure 1 The length distribution of the coding sequence (CDS) and predicted proteins by BLASTX and ESTScan software from the unigenes. A. Aligned CDS by BLASTX. B. Predicted proteins by BLASTX. C. Aligned CDS by ESTScan. D. Predicted proteins by ESTScan.

system evolution relationships of bacteria, algae and eukaryotes [32,33]. Overall, 19,888 of 73,084 (27.21\%) unigenes were assigned to the COG classification (Table 3 ). Since some of these unigenes were annotated with multiple COG functions, a total of 39,787 functional annotations were produced. Among the $25 \mathrm{COG}$ categories, the cluster for 'general functions prediction only' $(6,468,16.26 \%)$ associated with basic physiological and metabolic functions represented the largest group, followed by 'Transcription' (3,889, 9.77\%), 'Replication, recombination and repair' (3,326, 8.36\%), 'Post-translational modification, protein turnover, chaperones' $(2,974,7.47 \%)$, and 'Signal transduction mechanisms' $(2,937,7.38 \%)$, whereas only few unigenes were assigned to 'Extra cellular structures' and 'Nuclear structure' (Figure 4).

KEGG pathway database can facilitate to systematically understand the biological functions of genes in terms of networks [21,32]. To identify the biological pathways activated in radish roots, the assembled unigenes were annotated with KEGG Orthology (KO) numbers using
BLASTx alignments against KEGG with a cut-off E value of $10^{-5}$. A total of 33,567 unigenes were significantly matched in the database, and were assigned to 128 KEGG pathways. The result showed that the five largest pathway groups were metabolic pathways [ko01100, 7,391(22.02\%)], biosynthesis of secondary metabolites [ko01110, 3,363 (10.02\%)], plant hormone signal transduction [ko04075, 1,928(5.74\%)], plant-pathogen interactions [ko04626, 1,925 (5.74\%)] and RNA transport [ko30313, 1,285(3.83\%)] (Additional file 2). In metabolism categories, the biosynthesis of secondary metabolites represented the most predominant pathways, which were sorted into 13 subcategories including phenylpropanoid biosynthesis, glucosinolate biosynthesis, flavonoid biosynthesis, betalain biosynthesis and some others (Figure 5). These annotations of gene or protein names and descriptions, gene ontology terms, putative conserved domains, and potential metabolic pathways would provide a valuable resource for investigating specific processes, functions and pathways involved in radish taproot development. These genes involved in the 


\section{(A) E-value Distribution}

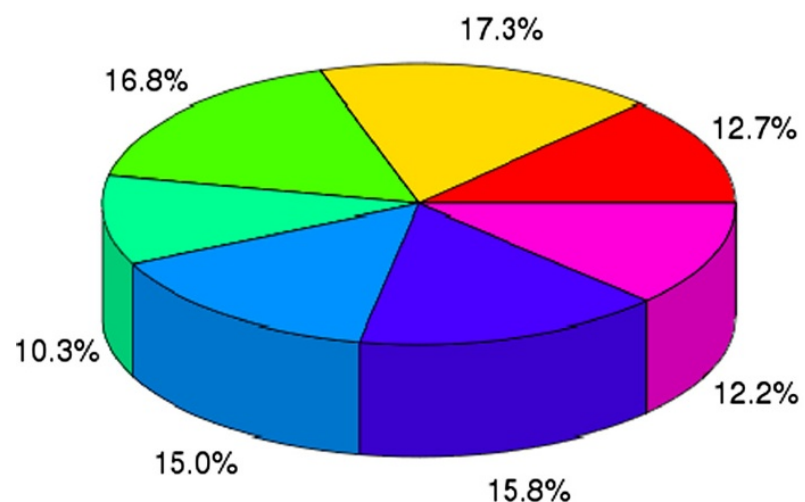

$$
\begin{aligned}
& =0 \\
& 0 \sim 1 \mathrm{e}-100 \\
& =1 \mathrm{e}-100 \sim 1 \mathrm{e}-60 \\
& =1 \mathrm{e}-60 \sim 1 \mathrm{e}-45
\end{aligned}
$$

\section{(B) Similarity Distribution}

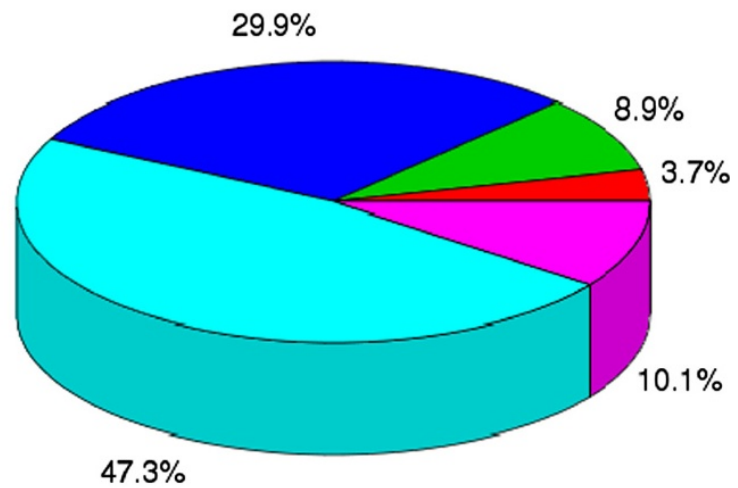

$47.3 \%$

\section{(C) Species Distribution}
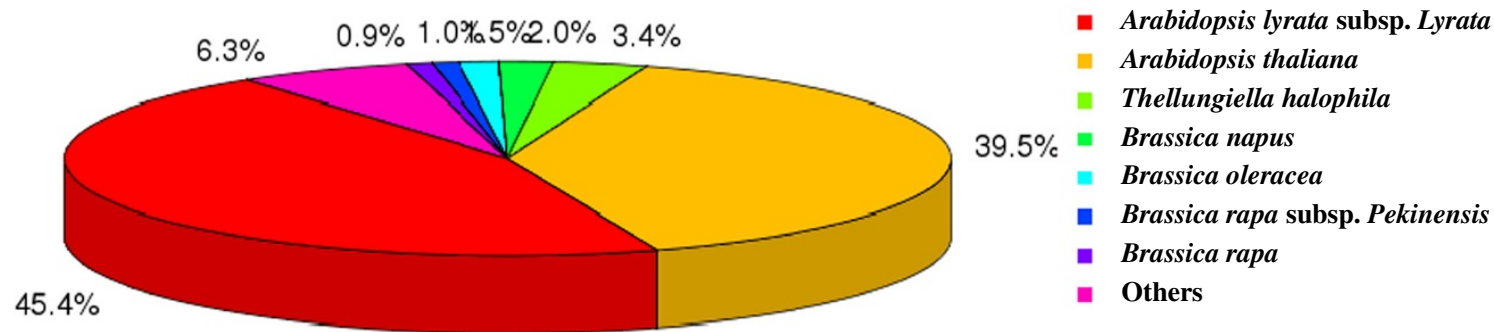

Figure 2 Characteristics of sequence homology of radish root BLASTED against NCBI non-redundant (NR) database. A. E-value distribution of BLAST hits for matched unigene sequences, using an E-value cutoff of $1.0 \mathrm{E}^{-5}$. B. Similarity distribution of top BLAST hits for each unigene. C. Species distribution of the top BLAST hits.

enrichment of secondary metabolite biosynthesis related pathways would greatly enhance the potential utilization of the radish root in nutrition and pharmacy.

\section{Identification of candidate genes involved in the} glucosinolate metabolism of radish

In the past decade, the main pathway of glucosinolate (GS) biosynthesis has been well understood in A. thaliana and $B$. rapa, and many critical genes have been successfully discovered and functionally characterized $[34,35]$. The biosynthesis of GS is generally divided into three independent phases: (i) amino acid side-chain elongation of selected precursor amino acids (only Met and Phe), (ii) core structure formation, (iii) and subsequent side-chain modification [36-38]. According to the currently accepted GS biosynthetic pathways in A. thaliana and B. rapa, a total of 94 unigenes in our transcriptome dataset were found to be homologous to the previously identified genes encoding all of the eight related enzymes of all three phases. The result indicated that this pathway was rather well conserved in Brassicaceae family. Furthermore, 14 unigenes were found to be homologous to the genes encoding myrosinase, which is a critical functional enzyme involved in the GS degradation (Figure 6 and Additional file 3). In most cases, more than one unique sequence was annotated as encoding the same enzyme. Such sequences may represent different fragments of a single transcript, different members of a gene family, or both $[17,39]$.

Initially, the parent amino acid is deaminated to form the corresponding 2-oxo acid by a branched-chain amino acid aminotransferase (BCAT, K00826, EC: 2.6.1.42). In $A$. 


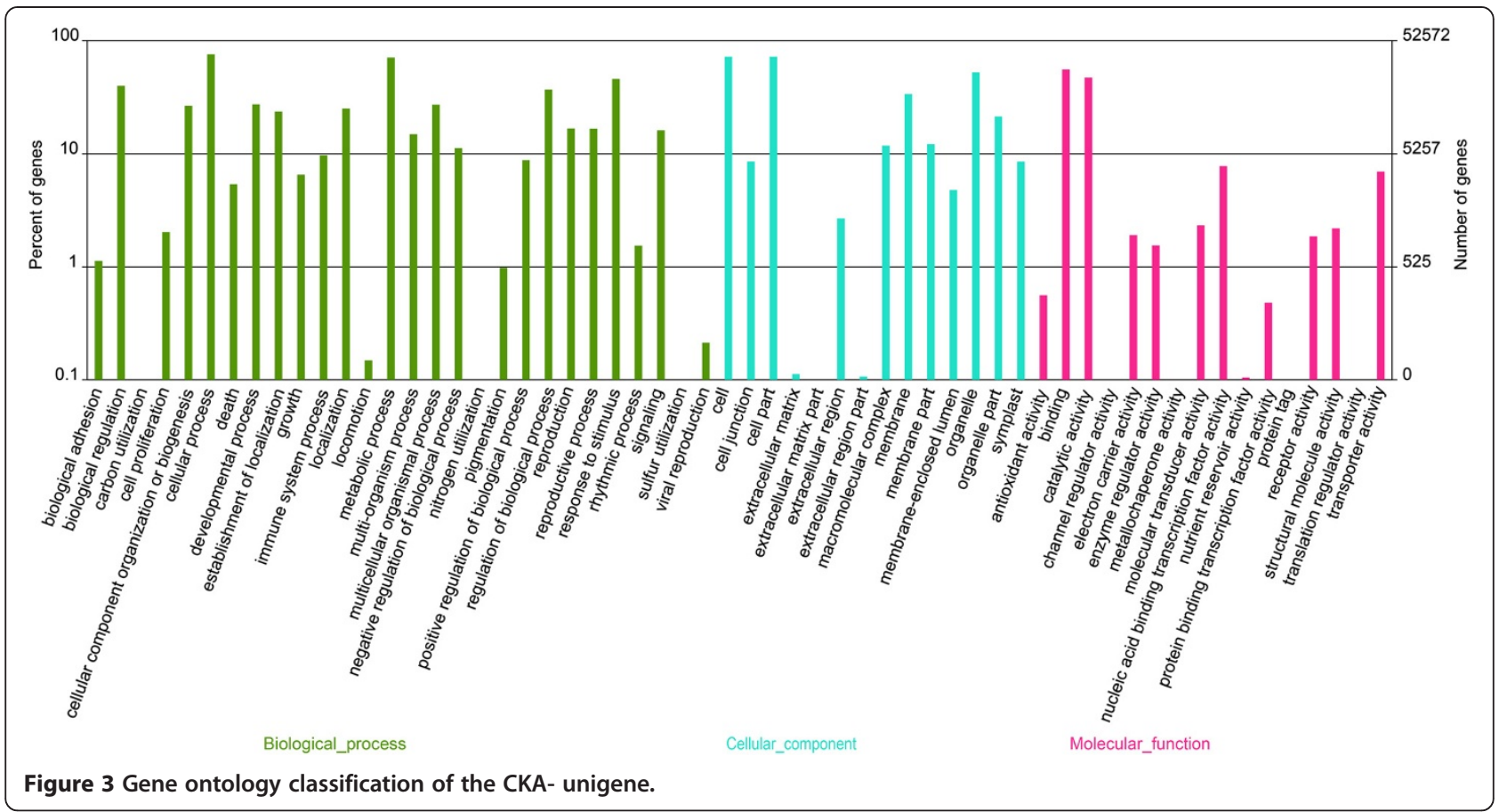

thaliana, there are seven genes encoding the BCATs, and it is known to be fairly well conserved [40]. In our annotated radish transcriptome unigene dataset, 17 sequences corresponding to five homologous BCAT genes (BCAT 2-5) were successfully identified. Subsequently, methylthioalkylmalate synthase (MAM, K15741, EC: 2.3.3.-) catalyzes 2-oxo acid condense with acetyl-CoA to yield a 2-oxo acid with one more methylene group $(-\mathrm{CH} 2-)$ than the starting compound. Hereupon, the elongated 2-oxo acid can enter the core glucosinolate

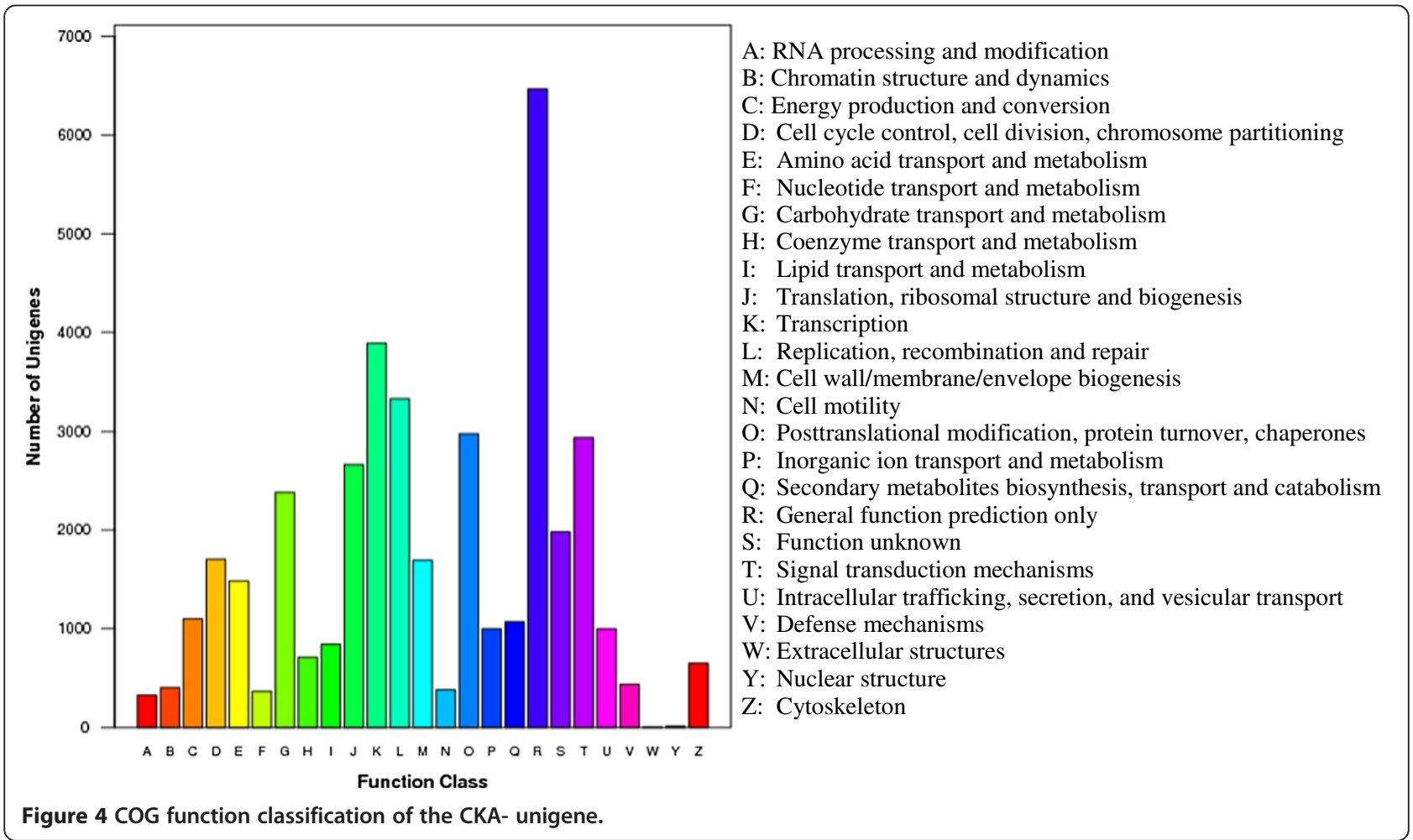




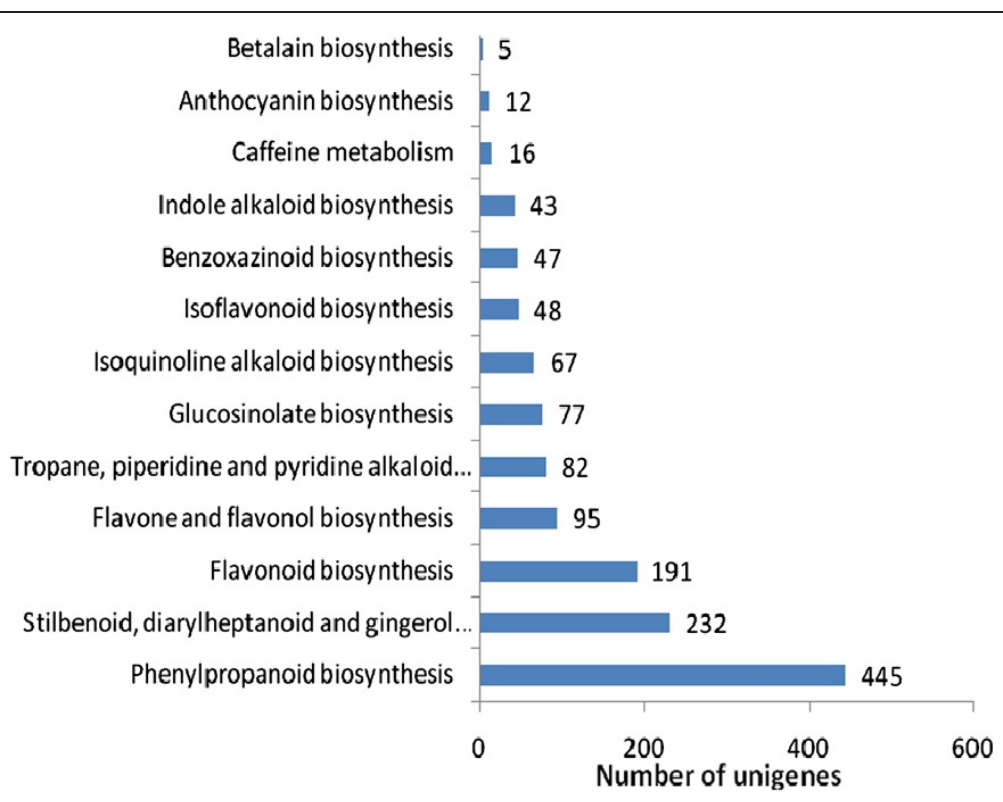

Figure $\mathbf{5}$ Classification based on categories of secondary metabolite biosynthesis.

Side-chain elongation

Side-chian elongated

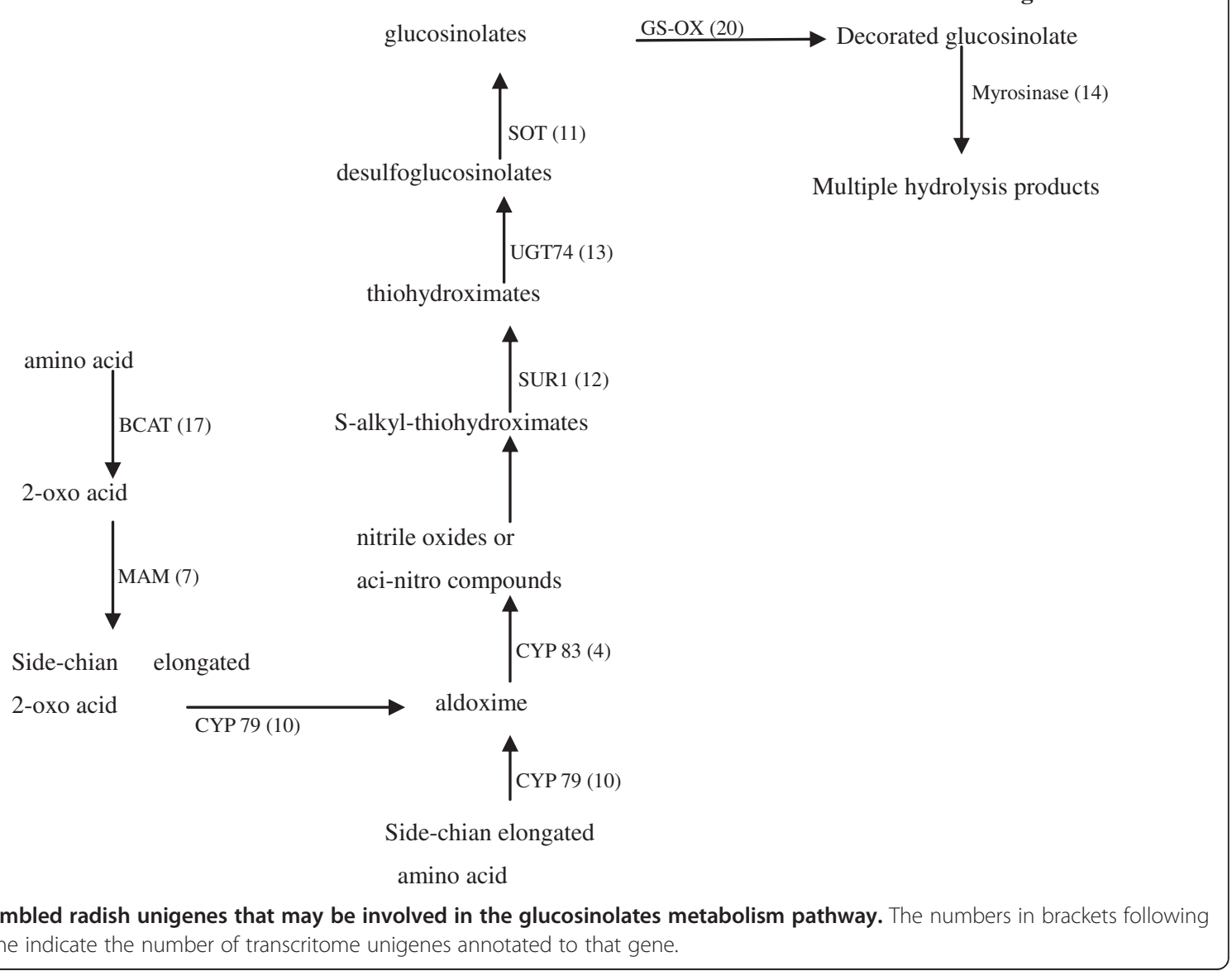

\section{Core structure formation}


structure pathway or proceed through another round of chain elongation. Seven sequences encoding MAM were discovered in our transcriptomic analysis.

The formation of primary glucosinolates involved in core structure biosynthesis is accomplished through five different biochemical steps that synthesize several intermediates. It begins with the oxidation of the precursor amino acids to aldoximes by cytochromes $\mathrm{P} 450$ belonging to the CYP79 family, which is composed of a number of catalytic subfamilies. Genome analyses have revealed that Arabidopsis contains seven different CYP79 genes (i.e. CYP79A1, B1, B2, B3, F1, F2 and F3) [41]. In the current study, ten unigene sequences were identified corresponding to the seven different genes with a high homology to CYP79s. All these seven gene members in the Arabidopsis genome were also identified in the radish transcriptome, which further confirmed the close relationship between these two species. Aldoximes are further oxidized to activated compounds (either nitrile oxides or aci-nitro compounds) by cytochromes P450 of the CYP83 family. Based on sequence similarities, four unigenes were identified corresponding to the two CYP83 genes (CYP83A1 and CYP83B1). The activated aldoximes are conjugated with cysteine as a sulfur donor to produce S-alkyl-thiohydroximates; however, it is not clear whether this conjugation is enzyme-mediated. The S-alkylthiohydroximate conjugates are converted to thiohydroximates by the C-S lyase SUPERROOT1 (SUR1, K11819) [42]. In the present study, 12 homolog sequences were discovered encoding SUR1. Thiohydroximates are in turn S-glucosylated by glucosyltransferases of the UGT74 family to form desulfoglucosinolates. Overall, 13 unigenes were identified as UGT74s including UGT74B1, C1, F1 and F2. The final step in the synthesis of the GS core structure was catalyzed by desulfoglucosinolate sulfotransferase (SOT, K11821). There are three close homologous SOT genes (SOT16, 17and18), which were identified in Arabidopsis to catalyze this reaction with a wide variety of desulfoglucosinolate substrates [43]. A total of 11 unigenes from our RNA-seq dataset were identified as SOTs including all three homologies found in Arabidopsis.

The initially produced parent glucosinolate from core structure is subject to a wide range of side chain modifications, which entail various kinds of reactions including oxidations, eliminations, alkylations, and esterifications. Kliebenstein et al. (2011) identified three genes responsible for side chain modification of aliphatic glucosinolates in Arabidopsis by QTL analyses [44], named GS-OX, GSAOP and GS-OH; and functionally characterized two genes including $A O P 2, A O P 3$ of the GS-AOP cluster. In this study, 20 unigenes ranging from $252 \mathrm{bp}$ to $1,921 \mathrm{bp}$ were homologous to the genes encoding GS-OX; however, the other genes corresponding to the modification of side chain could not be identified.
Upon plant damage, the GS can be degraded to a variety of hydrolysis products such as isothiocyanates, oxazolidine-2-thiones, nitriles, epithionitriles, and thiocyanates. The hydrolytic process is catalyzed by a Betathioglucoside glucohydrolase (myrosinase, EC 3.2.3.1, K01188). Until now, myrosinase genes have been isolated from many plant species such as turnip, A. thaliana and mustard, which indicated that these genes are encoded by a multigene family and were classified into four subtypes(MA, MB, MC and TGG) on the basis of amino acid sequences [45]. Additionally, two cDNA clones of myrosinase were isolated from radish seedlings, and both of them were identified as B type myrosinases [46]. In this study, 14 unigenes were found which were homologs of genes encoding myrosinase, and most of them were predicted as MB subtypes.

\section{Identification of genes involved in MYB transcription factors}

MYB transcription factors represent a family of proteins that include the conserved MYB DNA-binding domain, which can control diverse pathways and processes corresponding to plant secondary metabolism $[47,48]$. It was reported that many members of the MYB family could regulate the expression of related genes at the transcriptional level to control the process of GS metabolism in A. thaliana. For example, MYB28, 29 and 76 exerted a specific and coordinated control on the regulation of aliphatic GS biosynthesis, while MYB34, 51 and 122 could regulate the synthesis of indolic GS [49,50]. From our radish transcriptome analysis, a total of 257 unigenes were predicted to code MYB proteins including a large number of members (i.e., MYB 2, 3, 4, 25, 28, 29, 43, 47, 52, 56, 58, $65,69,73,78,95,103,108,121$, etc.) (Additional file 4). However, the specific function of the particular MYB member in GS metabolism of radish need to be further verified with functional genomics approach.

\section{Validation and expression analysis of genes involved in GS metabolism}

To check the quality of the assembly and annotation data from the Solexa sequencing, full-length cDNA sequences of eight selected genes from glucosinolate metabolism and regulation process were isolated by $\mathrm{T}$-A cloning with the Sanger method and compared with the assembled sequences. The length of these genes varied from 1,086 bp to 1,641 bp (Table 4). Overall, the assembled unigenes covered more than $95 \%$ of the corresponding full-length genes and two of them were predicted to contain the complete ORF. Additionally, the sequence variation was minimal (> 98\% pairwise identity), which validated the NGS-based RNA-seq procedures was reliable.

The qRT-PCR analysis was used to compare the dynamic expression patterns of four selected genes, RsBCAT4, 
Table 4 Sequence analyses of the eight putative radish genes involved in glucosinolate metabolism process

\begin{tabular}{cccccc}
\hline Gene & $\begin{array}{c}\text { Full-length } \\
\text { CDNA }\end{array}$ & Number & Coverage & $\begin{array}{c}\text { ORF } \\
\text { similarity }\end{array}$ & Gap \\
\hline RsBCAT4 & 1086 & 1 & $96.70 \%$ & $99.53 \%$ & $3.25 \%$ \\
RsCYP79F1 & 1623 & 2 & $98.58 \%$ & $99.94 \%$ & $0.73 \%$ \\
RSCYP83A1 & 1506 & 2 & $99.60 \%$ & $99.80 \%$ & $0.39 \%$ \\
RsSUR1 & 1371 & 3 & $100 \%$ & $99.93 \%$ & $5.30 \%$ \\
RsUGT74B1 & 1386 & 2 & $95.56 \%$ & $98.89 \%$ & $4.44 \%$ \\
RsGS-OX1 & 1380 & 2 & $100 \%$ & $99.80 \%$ & $0.39 \%$ \\
RsMYB28 & 1092 & 1 & $99.45 \%$ & $98.74 \%$ & $0.54 \%$ \\
RsMyr1 & 1647 & 1 & $99.81 \%$ & $99.52 \%$ & $0.24 \%$ \\
\hline
\end{tabular}

RsUGT74B1, RsGS-OX1 and RsMyr1, in different organs at three developmental stages. It was reported that several genes involved in the GS metabolism showed distinct spatiotemporal expression patterns in different species such as BCAT gene in A. thaliana [51], and Myr gene in $B$. napus [52], horseradish [53], and radish [46]. As shown in Figure 7, the expression of all these four genes in radish roots exhibited variations among different organs from different stages. RsBCAT4 was expressed weakly in root (including flesh and skin) at taproot thickening and mature stage, and the remaining samples showed inconspicuous changes. RsUGT74B1 exhibited higher expression in leaf and stem at seedling stage, and in stem at taproot thickening stage, whereas weaker expression was observed in root at all developmental stages. The expression of RsGS-OX1 in root decreased in the following order: seedling, taproot thickening, and mature stage. Obvious changes in the expression level of RsMyr1 were observed among organs at mature stages (fresh $>$ stem $>$ leaf $>$ skin), but exhibited inconspicuous variations at the other two stages.

\section{Conclusions}

In this study, NGS-based Illumina paired-end solexa sequencing platform was employed to characterize the fleshy taproot de novo transcriptome in radish. Approximately 66.11 million paired-end reads representing 73,084 unigenes with a N50 length of 1,095 bp, and a total length of $55.73 \mathrm{Mb}$ were obtained. A total of 67,305 unigenes were successfully annotated by blastx analysis using the publicly available protein database. It was revealed that the main genes activated in radish taproot, were predominately involved in basic physiological and metabolic processes, biosynthesis of secondary metabolites, signal transduction mechanisms, and other cellular components and molecular function related terms based on their matches in the GO, COG and KEGG databases. This study demonstrated that the Illumina paired-end sequencing technology is a fast and cost-effective method for novel gene discovery in non-model plant organisms. Furthermore, radish unigenes provided a comprehensive enough coverage to allow for the discovery of almost all genes known to be involved in GS metabolism and regulation related pathways. Our transcriptome dataset will serve as a valuable public platform to enhance the understanding of molecular mechanisms underlying biosynthesis and metabolism of the nutritional and flavor components during taproot formation. It would further facilitate the genetic improvement of major quality traits in radish breeding programs.

\section{Methods}

\section{Plant materials}

The radish (Raphanus sativus L.) advanced inbred line, 'NAU-RG', was used in this study. The surface-sterilized seeds were sown into soil in plastic pots and the seedlings were cultured in a growth chamber with $14 \mathrm{~h}$ light at $25^{\circ} \mathrm{C}$ and $10 \mathrm{~h}$ dark at $18^{\circ} \mathrm{C}$. For Solexa analysis and T-A cloning sequencing, taproots were sampled at three different developmental stages including seedling, taproot thickening, and mature stages. The subsamples of root, leaf and stem parts were collected at seedling, taproot thickening, and mature stages, respectively for qRTPCR verification (the skin and flesh at mature stage were separated). All samples were washed with distilled water, immediately frozen in liquid nitrogen and stored at $-80^{\circ} \mathrm{C}$ for RNA extraction.

\section{RNA extraction and Illumina sequencing}

Total RNA of the three taproot samples from different stages was isolated using the RNAprep pure Plant Kit (Tiangen Biotech Co., Ltd., China) according to the manufacturer's protocol. RNA samples were treated with RNasefree DNase I (Takara, Japan) to avoid DNA contamination. cDNA was prepared by equally pooling a total of $10 \mu \mathrm{g}$ of RNA from each of the taproot sample of three different developmental stages. The mixed root cDNA library named 'CKA' was constructed using an mRNA-seq assay for paired-end transcriptome sequencing, which was performed by the Beijing Genomics Institute (BGI, Shenzhen, China).

Poly(A) mRNA was enriched from total RNA by using Sera-mag Magnetic Oligo (dT) Beads (Thermo Fisher Scientific, USA) and then mRNA-enriched RNAs were chemically fragmented to short pieces using $1 \times$ fragmentation solution (Ambion, USA) for $2.5 \mathrm{~min}$ at $94^{\circ} \mathrm{C}$. These short fragments were taken as templates for firststrand cDNA synthesis using random hexamer-primer. The second-strand cDNA was generated using the SuperScript Double-Stranded cDNA Synthesis Kit (Invitrogen, USA). Short fragments were purified with QiaQuick PCR extraction kit and resolved with EB buffer for end repair and tailing A. Thereafter, the short fragments were connected with sequencing adapters, and the suitable fragments were selected for the PCR amplification 


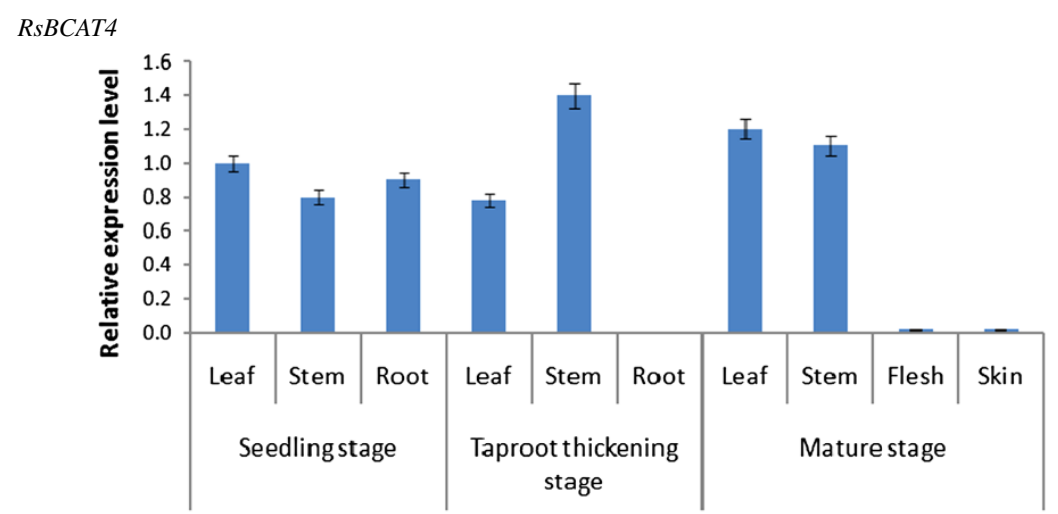

RsUGT74BI
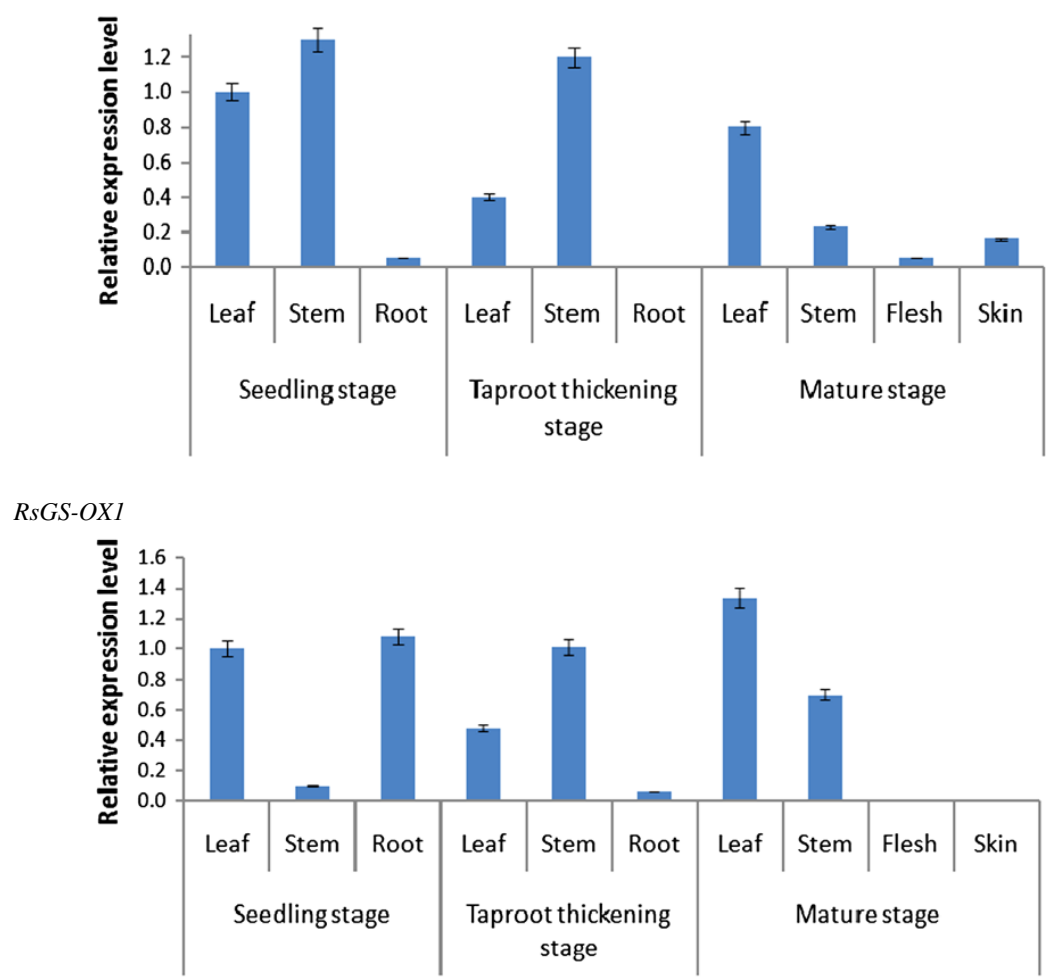

RsMyrl

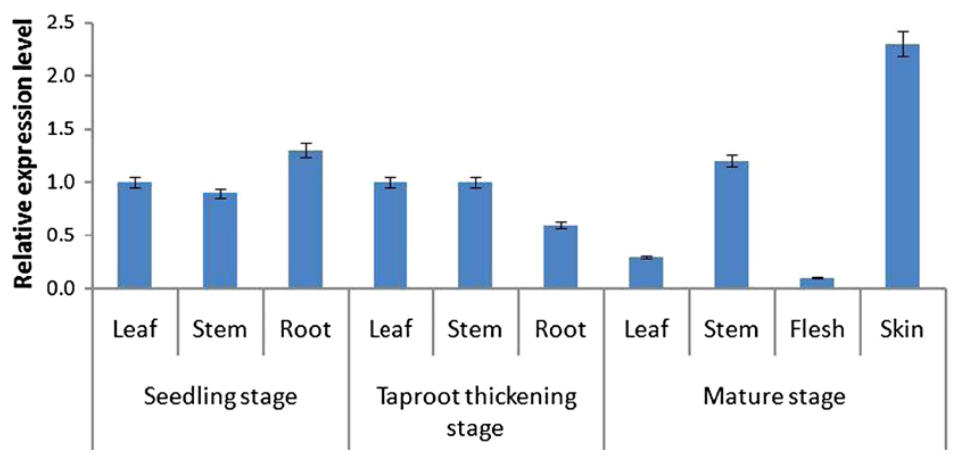

Figure 7 qRT-PCR expression analysis of four selected gene expression levels in different tissues during three developmental stages in radish. 
as templates after agarose gel electrophoresis. Finally, the library was sequenced using Illumina $\mathrm{HiSeq}^{\mathrm{TM}} 2000$.

\section{Raw sequence processing and de novo assembly}

Raw reads generated by Illumina Hiseq ${ }^{\mathrm{TM}} 2000$ were initially processed to get clean reads. Then, all the clean reads were assembled using a de novo assembly program Trinity [54]. Firstly, clean reads with a certain length of overlap were combined to form longer contiguous sequences (contigs), and then these reads were mapped back to the contigs. The distance and relation among these contigs was calculated based on paired-end reads, which enabled the detection of contigs from the same transcript and also the calculation of distances among these contigs. Finally, the contigs were further assembled using Trinity, and the contigs that could not be extended on either end were defined as unique transcripts. Additionally, the unigenes were divided into two classes by gene family clustering. The prefix $\mathrm{CL}$ was given to the clusters following the cluster id. Several unigenes with over $70 \%$ similarity were included from one cluster while from the other group the unigenes selected were singletons, for which the prefix unigene was used.

\section{Functional annotation and classification of the assembled transcripts}

All of the assembled transcripts were compared with the publicly available protein databases including NCBI nonredundant protein $(\mathrm{Nr})$, Gene Ontology (GO), Clusters of Orthologous Groups (COGs), Swiss-Prot protein and the Kyoto Encyclopedia of Genes and Genomes (KEGG), using the BLASTx analysis with a cut-off $E$ value of $10^{-5}$. The best alignments were used to identify sequence direction and to predict the coding regions of the assembled unigenes. If the results from different databases conflicted with each other, a priority order of nr, Swiss-Prot, KEGG and COG was followed. When a unigene happened to be unaligned to none of the above databases, software ESTScan was introduced to decide its sequence direction [55]. For the nr annotations, the BLAST2GO program was used to get GO annotations of unique assembled transcripts for describing biological processes, molecular functions, and cellular components [56]. After getting GO annotations for each transcript, WEGO software [57] was used to conduct $\mathrm{GO}$ functional classification for understanding the distribution of gene functions at the macroscopic level.

\section{Gene validation by T-A cloning and sequencing}

Specific PCR primers of the eight selected genes (Additional file 5) were designed corresponding to the conserved region of radish EST sequences from radish cDNA library [58]. PCR was performed in a total volume of $25 \mu \mathrm{l}$ containing $2.0 \mathrm{mmol} / \mathrm{L} \mathrm{Mg}^{2+}, 0.15 \mathrm{mmol} / \mathrm{L}$ dNTPs, $0.4 \mathrm{mmol} / \mathrm{L}$ of each primer, $0.8 \mathrm{U}$ Taq DNA polymerase (TAKARA) and 15 ng cDNA with the following conditions: an initial denaturation step at $94^{\circ} \mathrm{C}$ for $1 \mathrm{~min}, 35$ cycles at $94^{\circ} \mathrm{C}$ for $50 \mathrm{~s}, 56^{\circ} \mathrm{C}$ for $50 \mathrm{~s}$, and $72^{\circ} \mathrm{C}$ for $90 \mathrm{~s}$, a final extension at $72^{\circ} \mathrm{C}$ for $10 \mathrm{~min}$ and hold at $4^{\circ} \mathrm{C}$. The PCR products were separated and ligated into the pMD18-T vector (Takara Bio Inc., China), and then transformed into E. coli DH5 $\alpha$. Positive clones were sequenced with ABI 3730 (Applied Biosystems, USA).

\section{Quantitative real-time PCR (qRT-PCR) analysis}

Quantitative real-time PCR was performed on a MyiQ Real-Time PCR Detection System (Bio-Rad) platform using the SYBR Green Master ROX (Roche, Japan) following the manufacturer's instructions. Primers were designed using Beacon Designer 7.0 software, and Actin2/7 $(A C T)$ (Additional file 6) was selected as the internal control gene [59]. Amplification was achieved by a PCR program having a first denaturation step at $95^{\circ} \mathrm{C}$ for $5 \mathrm{~min}$, then 40 cycles of denaturation at $95^{\circ} \mathrm{C}$ for $5 \mathrm{~s}$, followed by annealing and extension at $58^{\circ} \mathrm{C}[30,59]$. The relative expression levels of the selected transcripts were normalized to $A C T$ gene and calculated using the $2^{-\Delta \Delta C t}$ method. All reactions were performed in three replicates, and the data were analyzed using the Bio-Rad CFX Manager software.

\section{Availability of supporting data}

The RNA sequence dataset supporting the results of this article is available in the [NCBI Sequence Read Archive] repository, [SRX316199 and http://www.ncbi.nlm.nih.gov/ $\mathrm{sra} /]$.

\section{Additional files}

\section{Additional file 1: Length frequency distribution of contigs and unigenes obtained from de novo assembly.}

Additional file 2: KEGG pathways of the assembled transcripts.

Additional file 3: Candidate genes involved in glucosinolate metabolism of radish.

Additional file 4: Candidate genes involved in MYB transcript factors.

Additional file 5: Primers used for T-A cloning and sequencing. Additional file 6: Primers used for qRT-PCR analysis.

\section{Competing interests}

The authors declare that they have no competing interests for this research.

\section{Authors' contributions}

WY designed the experiments and drafted the manuscript. PY and LZ planted radish seedlings, collected tissues and prepared the mRNA library for Solexa sequencing. $\mathrm{ZL}, \mathrm{XL}$ and $\mathrm{YR}$ participated in the design of the study and performed the statistical analysis. ZX and GY participated in the sequence alignment and gene validation and expression analysis. $\mathrm{LL}$ conceived of the study, and participated in its design and coordination and helped to draft the manuscript. All authors read and approved the final manuscript. 


\section{Acknowledgements}

This work was in part supported by grants from the National Key Technology R \& D Program of China (2012BAD02B01, 2011GB23600006), NSFC (31171956, 31372064), Key Technology R \& D Program of Jiangsu Province (BE2010328), FRFCU(KYZ201209), JASTIF [CX (12)2006] and the PAPD.

\section{Author details}

${ }^{1}$ National Key Laboratory of Crop Genetics and Germplasm Enhancement, College of Horticulture, Nanjing Agricultural University, Nanjing 210095, P.R. China. ${ }^{2}$ Engineering Research Center of Horticultural Crop Germplasm Enhancement and Utilization, Ministry of Education of P.R. China, College of Horticulture, Nanjing Agricultural University, Nanjing 210095, P.R. China. ${ }^{3}$ Institute of Vegetable Crops, Wenzhou Academy of Agricultural Sciences; Wenzhou Vocation College of Science \& Technology, Wenzhou 325014, P.R. China. ${ }^{4}$ Department of Plant Sciences, North Dakota State University, Fargo, ND 58108, USA.

Received: 17 June 2013 Accepted: 14 November 2013

Published: 27 November 2013

\section{References}

1. Wang L, He Q: Chinese radish. Beijing, China: Scientific and Technical Document Publishing House; 2005.

2. Curtis IS: Genetic transformation of radish (Raphanus sativus L.) by floraldipping. In Transgenic Crops of the World. Edited by Curtis IS. Dordrecht: Kluwer Academic Publishers; 2004:271-280.

3. Khanum F, Swamy MS, Krishna KS, Santhanam K, Viswanathan K: Dietary fiber content of commonly fresh and cooked vegetables consumed in India. Plant Foods Hum Nutr 2000, 55(3):207-218.

4. Curtis IS: Genetic engineering of radish: current achievements and future goals. Plant Cell Rep 2011, 30(5):733-744.

5. Vardhini BV, Sujatha E, Rao SSR: Studies on the effect of brassinosteroids on the qualitative changes in the storage roots of radish. Bulg J Agric SCi 2012, 18(1):63-69.

6. Hara M, Ito F, Asai T, Kuboi T: Variation in amylase activities in radish (Raphanus sativus) cultivars. Plant Foods Hum Nutr 2009, 64(3):188-192.

7. Hara M, Sawada T, Ito A, Ito F, Kuboi T: A major $\beta$-amylase expressed in radish taproots. Food Chem 2009, 114(2):523-528.

8. Ishida M, Kakizaki T, Ohara T, Morimitsu Y: Development of a simple and rapid extraction method of glucosinolates from radish roots. Breed Sci 2011, 61(2):208-211.

9. Holst B, Williamson G: A critical review of the bioavailability of glucosinolates and related compounds. Nat Prod Rep 2004, 21(3):425-447.

10. Usuda $\mathrm{H}$ : Effects of growth under elevated $\mathrm{CO}_{2}$ on the capacity of photosynthesis in two radish cultivars differing in capacity of storage root. Plant Prod Sci 2004, 7(4):377-385

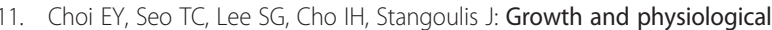
responses of Chinese cabbage and radish to long-term exposure to elevated carbon dioxide and temperature. Hortic Environ Biotechnol 2011, 52(4):376-386

12. Samuolienè G, Sirtautas R, Brazaitytè A Sakalauskaitè J, Sakalauskienè S, Duchovskis $P$ : The impact of red and blue light-emitting diode illumin ation on radish physiological indices. Cent Eur J Biol 2011, 6(5):821-828.

13. Wang S, Wang X, He Q, Liu X, Xu W, Li L, Gao J, Wang F: Transcriptome analysis of the roots at early and late seedling stages using Illumina paired-end sequencing and development of EST-SSR markers in radish. Plant Cell Rep 2012, 31(8):1437-1447.

14. Sherman BT, Lempicki RA: Bioinformatics enrichment tools: paths toward the comprehensive functional analysis of large gene lists. Nucleic Acids Res 2009, 37(1):1-13.

15. Varshney RK, Nayak SN, May GD, Jackson SA: Next-generation sequencing technologies and their implications for crop genetics and breeding. Trends Biotechnol 2009, 27(9):522-530.

16. Ward JA, Ponnala $L$, Weber CA: Strategies for transcriptome analysis in nonmodel plants. Am J Bot 2012, 99(2):267-276.

17. Hyun TK, Rim Y, Jang H-J, Kim CH, Park J, Kumar R, Lee S, Kim BC, Bhak J, Nguyen-Quoc B: De novo transcriptome sequencing of Momordica cochinchinensis to identify genes involved in the carotenoid biosynthesis. Plant Mol Biol 2012, 79(4-5):413-427.
18. Huang HH, Xu LL, Tong ZK, Lin EP, Liu QP, Cheng LJ, Zhu MY: De novo characterization of the Chinese fir (Cunninghamia lanceolata) transcriptome and analysis of candidate genes involved in cellulose and lignin biosynthesis. BMC Genomics 2012, 13(1):648.

19. Shi CY, Yang H, Wei CL, Yu O, Zhang ZZ, Jiang CJ, Sun J, Li YY, Chen Q, Xia $\mathrm{T}$ : Deep sequencing of the Camellia sinensis transcriptome revealed candidate genes for major metabolic pathways of tea-specific compounds. BMC Genomics 2011, 12(1):131.

20. Li H, Dong Y, Yang J, Liu X, Wang Y, Yao N, Guan L, Wang N, Wu J, Li X: De novo transcriptome of safflower and the identification of putative genes for oleosin and the biosynthesis of flavonoids. PLoS One 2012, 7(2):e30987.

21. Hua WP, Zhang Y, Song J, Zhao LJ, Wang ZZ: De novo transcriptome sequencing in Salvia miltiorrhiza to identify genes involved in the biosynthesis of active ingredients. Genomics 2011, 98(4):272-279.

22. Liu S, Li W, Wu Y, Chen C, Lei J: De novo transcriptome assembly in chili pepper (Capsicum frutescens) to identify genes involved in the biosynthesis of capsaicinoids. PLoS One 2013, 8(1):e48156.

23. Ishii G: Glucosinolate in Japanese radish, Raphanus sativus L. Jpn Agric Res O 1991, 24:273-279.

24. Ishida M, Nagata M, Ohara T, Kakizaki T, Hatakeyama K, Nishio T: Small variation of glucosinolate composition in Japanese cultivars of radish (Raphanus sativus L.) requires simple quantitative analysis for breeding of glucosinolate component. Breed Sci 2012, 62(1):63.

25. Zou Z, Ishida M, Li F, Kakizaki T, Suzuki S, Kitashiba H, Nishio T: QTL analysis using SNP markers developed by next-generation sequencing for identification of candidate genes controlling 4-methylthio-3-butenyl glucosinolate contents in roots of radish, Raphanus sativus L. PLoS One 2013 8(1):e53541

26. Liang C, Liu X, Yiu S-M, Lim BL: De novo assembly and characterization of Camelina sativa transcriptome by paired-end sequencing. BMC Genomics 2013, 14(1):146.

27. Huang LL, Yang $X$, Sun $P$, Tong W, Hu SQ: The first Illumina-based de novo transcriptome sequencing and analysis of safflower flowers. PLoS One 2012, 7(6):e38653.

28. Xie F, Burklew CE, Yang Y, Liu M, Xiao P, Zhang B, Qiu D: De novo sequencing and a comprehensive analysis of purple sweet potato (Impomoea batatas L.) transcriptome. Planta 2012, 236(1):101-113.

29. Logacheva M, Kasianov A, Vinogradov D, Samigullin T, Gelfand M, Makeev V Penin A: De novo sequencing and characterization of floral transcriptome in two species of buckwheat (Fagopyrum). BMC Genomics 2011, 12(1):30.

30. Dang ZH, Zheng LL, Wang J, Gao Z, Wu SB, Qi Z, Wang YC: Transcriptomic profiling of the salt-stress response in the wild recretohalophyte Reaumuria trigyna. BMC Genomics 2013, 14(1):29.

31. Harris M, Clark J, Ireland A, Lomax J, Ashburner M, Foulger R, Eilbeck K, Lewis S, Marshall B, Mungall C: The Gene Ontology (GO) database and informatics resource. Nucleic Acids Res 2004, 32:258-261.

32. Kanehisa M, Goto S: KEGG: kyoto encyclopedia of genes and genomes. Nucleic Acids Res 2000, 28(1):27-30.

33. Dutkowski J, Tiuryn J: Identification of functional modules from conserved ancestral protein-protein interactions. Bioinformatics 2007, 23(13):i149-i158.

34. Zang YX, Kim HU, Kim JA, Lim MH, Jin M, Lee SC, Kwon SJ, Lee SI, Hong JK Park TH: Genome-wide identification of glucosinolate synthesis genes in Brassica rapa. FEBS J 2009, 276(13):3559-3574.

35. Wang $H$, Wu J, Sun S, Liu B, Cheng F, Sun R, Wang X: Glucosinolate biosynthetic genes in Brassica rapa. Gene 2011, 487(2):135.

36. Wittstock U, Halkier B: Glucosinolate research in the Arabidopsis era. Trends Plant Sci 2002, 7(6):263-270.

37. Yang G, Gao Y-T, Shu X-O, Cai Q, Li G-L, Li H-L, Ji B-T, Rothman N, Dyba M, Xiang Y-B: Isothiocyanate exposure, glutathione S-transferase polymorphisms, and colorectal cancer risk. Am J Clin Nutr 2010, 91(3):704-711.

38. Sønderby IE, Geu-Flores F, Halkier BA: Biosynthesis of glucosinolates-gene discovery and beyond. Trends Plant Sci 2010, 15(5):283-290.

39. Sun C, Li Y, Wu Q, Luo H, Sun Y, Song J, Lui E, Chen S: De novo sequencing and analysis of the American ginseng root transcriptome using a GS FLX Titanium platform to discover putative genes involved in ginsenoside biosynthesis. BMC Genomics 2010, 11(1):262

40. Diebold R, Schuster J, Däschner K, Binder S: The branched-chain amino acid transaminase gene family in Arabidopsis encodes plastid and mitochondrial proteins. Plant Physio/ 2002, 129(2):540-550.

41. Grubb CD, Abel S: Glucosinolate metabolism and its control. Trends Plant Sci 2006, 11(2):89-100 
42. Mikkelsen MD, Naur P, Halkier BA: Arabidopsis mutants in the C-S lyase of glucosinolate biosynthesis establish a critical role for indole-3acetaldoxime in auxin homeostasis. Plant J 2004, 37(5):770-777.

43. Piotrowski M, Schemenewitz A, Lopukhina A, Müller A, Janowitz T, Weiler EW, Oecking C: Desulfoglucosinolate sulfotransferases from Arabidopsis thaliana catalyze the final step in the biosynthesis of the glucosinolate core structure. J Biol Chem 2004, 279(49):50717-50725.

44. Kliebenstein DJ, Gershenzon J, Mitchell-Olds T: Comparative quantitative trait loci mapping of aliphatic, indolic and benzylic glucosinolate production in Arabidopsis thaliana leaves and seeds. Genetics 2001, 159(1):359-370.

45. Rask L, Andréasson E, Ekbom B, Eriksson S, Pontoppidan B, Meijer J: Myrosinase: gene family evolution and herbivore defense in Brassicaceae. Plant Mol Biol 2000, 42(1):93-113.

46. Hara M, Fujii Y, Sasada Y, Kuboi T: cDNA cloning of radish (Raphanus sativus) myrosinase and tissue-specific expression in root. Plant Cell Physiol 2000, 41(10):1102-1109.

47. Jin $\mathrm{H}$, Martin $\mathrm{C}$ : Multifunctionality and diversity within the plant MYBgene family. Plant Mol Biol 1999, 41(5):577-585.

48. Laitinen RA, Ainasoja M, Broholm SK, Teeri TH, Elomaa P: Identification of target genes for a MYB-type anthocyanin regulator in Gerbera hybrida. J Exp Bot 2008, 59(13):3691-3703.

49. Gigolashvili T, Engqvist M, Yatusevich R, Müller C, Flügge UI: HAG2/MYB76 and HAG3/MYB29 exert a specific and coordinated control on the regulation of aliphatic glucosinolate biosynthesis in Arabidopsis thaliana. New Phytol 2008, 177(3):627-642.

50. Sønderby IE, Burow M, Rowe HC, Kliebenstein DJ, Halkier BA: A complex interplay of three R2R3 MYB transcription factors determines the profile of aliphatic glucosinolates in Arabidopsis. Plant Physiol 2010, 153(1):348-363.

51. Schuster J, Binder S: The mitochondrial branched-chain aminotransferase (AtBCAT-1) is capable to initiate degradation of leucine, isoleucine and valine in almost all tissues in Arabidopsis thaliana. Plant Mol Biol 2005, 57(2):241-254.

52. McCully ME, Miller C, Sprague SJ, Huang CX, Kirkegaard JA: Distribution of glucosinolates and sulphur-rich cells in roots of field-grown canola (Brassica napus). New Phytol 2008, 180(1):193-205.

53. Li X, Kushad MM: Purification and characterization of myrosinase from horseradish (Armoracia rusticana) roots. Plant Physiol Biochem 2005, 43(6):503-511.

54. Grabherr MG, Haas BJ, Yassour M, Levin JZ, Thompson DA, Amit I, Adiconis $X$, Fan L, Raychowdhury R, Zeng Q: Full-length transcriptome assembly from RNA-Seq data without a reference genome. Nat Biotechnol 2011, 29(7):644-652

55. Iseli C, Jongeneel CV, Bucher P: ESTScan: a program for detecting, evaluating, and reconstructing potential coding regions in EST sequences. Proc Int Conf Intell Syst Mol Biol 1999, 1999:138-148.

56. Conesa A, Götz S, García-Gómez JM, Terol J, Talón M, Robles M: Blast2GO: a universal tool for annotation, visualization and analysis in functional genomics research. Bioinformatics 2005, 21(18):3674-3676.

57. Ye J, Fang L, Zheng H, Zhang Y, Chen J, Zhang Z, Wang J, Li S, Li R, Bolund L: WEGO: a web tool for plotting GO annotations. Nucleic Acids Res 2006, 34(suppl 2):293-297.

58. Jiang LN, Wang L, Liu LW, Zhu XW, Zhai LL, Gong YQ: Development and characterization of CDNA library based novel EST-SSR marker in radish (Raphanus sativus L.). Sci Hortic 2012, 140:164-172.

59. Xu Y, Zhu X, Gong Y, Xu L, Wang Y, Liu L: Evaluation of reference genes for gene expression studies in radish (Raphanus sativus $L$.) using quantitative real-time PCR. Biochem Biophys Res Commun 2012, 424:398-403.

doi:10.1186/1471-2164-14-836

Cite this article as: Wang et al: De novo transcriptome sequencing of radish (Raphanus sativus $\mathrm{L}$.) and analysis of major genes involved in glucosinolate metabolism. BMC Genomics 2013 14:836.

\section{Submit your next manuscript to BioMed Central and take full advantage of:}

- Convenient online submission

- Thorough peer review

- No space constraints or color figure charges

- Immediate publication on acceptance

- Inclusion in PubMed, CAS, Scopus and Google Scholar

- Research which is freely available for redistribution

Submit your manuscript at www.biomedcentral.com/submit 